Cite this: CrystEngComm, 2013, 15, 2275

Received 2nd October 2012,

Accepted 28th November 2012

DOI: $10.1039 / c 2 c e 26607 e$

www.rsc.org/crystengcomm

\section{The development of the depletion zone during ceiling crystallization: phase shifting interferometry and simulation results $\dagger$}

\author{
Alaa Adawy, ${ }^{* a}$ Kess Marks, ${ }^{a}$ Willem J. de Grip, ${ }^{b}$ Willem J. P. van Enckevort*a \\ and Elias Vlieg ${ }^{a}$
}

\begin{abstract}
The growth of high quality protein crystals is essential for the determination of their structure. This process is governed by many physical factors such as mass transport and solution flow. The quality of the crystals is usually better under diffusion-limited growth conditions, where a depleted zone of the solution encapsulates the crystal. We developed a Mach-Zehnder-based phase shifting interferometer coupled to image processing software to study the concentration gradients which develop around a crystal during its growth or dissolution. The depletion zones and the diffusion boundary layers around growing and dissolving $\mathrm{KH}_{2} \mathrm{PO}_{4}$ crystals are monitored and processed by a MATLAB based algorithm. Our main emphasis was to analyze the ceiling crystallization conditions in which the crystal is placed at the very top of the growth cell and therefore the solute transport is largely diffusion-limited. The experimental results are compared with simulations using finite element-based numerical calculations. The combined results clearly demonstrate the positive effect of the ceiling crystallization approach on crystal growth.
\end{abstract}

\section{Introduction}

Crystal growth takes place at the crystal-solution interface, involving both solute mass transport and growth kinetics. As a consequence, the solution in the direct vicinity of the crystal generally undergoes a decrease in density. ${ }^{1}$ This is due to the incorporation of solutes into the crystal from the surrounding solution and, to a lesser extent, the release of thermal energy. This low density portion of the solution can either settle around the growing crystal (diffusion-limited growth), or it can undergo a rapid exchange with its surroundings by gravityinduced natural convection.

From the two regimes, diffusion-limited crystal growth is an amenity in obtaining high quality protein crystals. ${ }^{2}$ Many methods were developed in order to provide convection-free growth conditions such as the counter diffusion method, ${ }^{3}$ exploiting magnetic fields to counter the gravitational field, ${ }^{4}$ or space microgravity experiments. ${ }^{5}$ In these methods and after nucleation, crystal growth induces the development of a depletion zone, which is not disturbed by natural convection.

\footnotetext{
${ }^{a}$ Institute for Molecules and Materials, Radboud University Nijmegen, The

Netherlands.E-mail: a.adawy@science.ru.nl; W.vanEnckevort@science.ru.nl; Fax: +31 (0)24 3653067; Tel: +31 (0)24 3653365

${ }^{b}$ Department of Biochemistry, Nijmegen Centre for Molecular Life Sciences, Radboud University Nijmegen Medical Centre, The Netherlands

$\dagger$ Electronic supplementary information (ESI) available: Table of the physical constants used in the numerical simulations, movie showing the interferograms of the growth of tetragonal HEWL crystals during the first day after the onset of the experiment. See DOI: 10.1039/c2ce26607e
}

The developing concentration gradient limits the mass transport of growth units towards the growing crystal to be solely diffusive. This has a positive impact on the crystal quality because the growth rate slows down, allowing the crystal to embed growth units at the right orientation on the crystal surface. In addition, sedimentation of small crystals and incorporation of impurities are reduced. ${ }^{6}$

In a previous paper it was shown that diffusive crystal growth can also be accomplished in a simple terrestrial setup by using an upside-down geometry which exploits gravity. ${ }^{7}$ In this setup, the crystals at the top (ceiling) of a growth cell grow in a diffusion-limited crystallization environment. Ceiling crystals grow slowly and because of the "upside-down" geometry, the growing crystals are not affected by sedimentation. This eventually results in better diffracting crystals with lower impurity content when compared with those grown at the bottom of the same setup. ${ }^{8}$ This was clearly demonstrated by statistical analysis, which showed that the resolution limit of ceiling crystals (diffusive growth) is significantly improved over that of the batch crystals (convective growth) at a high confidence level. Quantitative experimental data are beyond the scope of the present paper and are presented elsewhere. ${ }^{8}$

To examine the different hydrodynamics during ceiling and batch crystallization, we used tetragonal crystals of potassium dihydrogen phosphate (KDP) as a model compound. ${ }^{9}$ We used aqueous KDP solutions of either $10 \%$ and $20 \%$ supersaturation or $10 \%$ and $20 \%$ undersaturation level to study both the growth and the dissolution processes. Emphasis is laid on 
monitoring the development of the depletion zone during ceiling growth.

To visualize the buoyancy-driven convection plumes (batch growth) or the development of the depletion zone (ceiling growth), we relied on optical interferometry to map the solute concentration profile in the solution. ${ }^{10}$ The concentration profile around the growing crystal can be monitored as a local change in the refractive index of the crystallization solution, which is a function of solute concentration. Interferometric techniques provide a route to map these differences in the refractive index in terms of changes in the optical path length. ${ }^{11}$ We designed a Mach-Zehnder-based phase shifting interferometer and used a five frame algorithm in order to study the changes in the spatial distribution of solute concentration, which take place during the growth or dissolution of KDP. These observations are supplemented by finite element-based numerical simulations for the same dissolution or growth conditions. Some conclusions from this study are validated by comparing the morphological quality of ceiling and batch grown hen egg-white lysozyme (HEWL) crystals grown in cells of different sizes.

\section{Concentration profile measurements}

\section{Mach-Zehnder phase shifting interferometer (PSI)}

To map the concentration profiles, we used a Mach-Zehnder phase shifting interferometer (Fig. 1). This two-beam interferometer for transmitted light favours high accuracy and easy quantitative interpretation. The incident monochromatic laser beam is split into two coherent beams, one of which passes the transparent specimen, i.e. the growth cell which contains the crystal and the solution, and the other acts as a reference beam. The two beams superimpose after travelling approximately the same geometrical distance resulting in an interference pattern $I(x, y)$, which embeds the information of the amplitude and phase, ${ }^{12}$ such that:

$$
I(x, y)=I_{\mathrm{o}}+I_{\mathrm{r}}+2 \sqrt{I_{\mathrm{o}} I_{\mathrm{r}}} \cos \varphi(x, y)
$$

where $I, I_{\mathrm{r}}$ and $I_{\mathrm{o}}$ are the intensities of the resultant, reference and object beam, respectively and $\phi(x, y)$ is the position dependent phase difference of both beams.

In order to retrieve the object phase information, physical shifts are introduced between the two beams. This is accomplished by recording a sequence of interferograms, each with the object beam displaced by a phase shift of $\pi / 2(\propto$ $\lambda / 4)$. In this way, the phase information can be retrieved by using the appropriate algorithm to process the consecutively recorded interferograms. Here, we adopted the 5B-frame algorithm introduced by Schmidt and Creath, ${ }^{13}$ because it is the most efficient in obtaining a high accuracy. In this case, the total phase shift after the acquisition of the five interferograms is $2 \pi$, and the algorithm yields

$$
\varphi(x, y)=-\tan ^{-1}\left[\frac{3 I_{2}-3 I_{3}-I_{4}+I_{5}}{I_{1}-I_{2}-3 I_{3}+3 I_{4}}\right]
$$

with $I_{j}=1-5$ the intensity of the five interferograms at point ( $x$, $y$ ). The phase information reveals the spatial changes in the refractive index and thus, the concentration profile. Based on this algorithm, a MATLAB script was written, which combines the five $\pi / 2$-phase shifted interferograms and a mask frame (representing the crystal body) to give a discontinuous phase profile disregarding the body of the crystal. The discontinuities in the calculated phase profile, owing to the nature of the arctangent function, introduce errors in the calculated phase. In order to remove these errors, we developed a correction script, which compares the phase value at each pixel with that of the previous neighbouring vertical or horizontal pixel. If the difference is $<-3 \pi / 4, \pi$ is added to the pixel and if this difference is $>3 \pi / 4, \pi$ is subtracted from this pixel, otherwise the phase value is not changed. Finally, the corrected phase profile is used to compute the concentration profile. This is done by exploiting the relation between the refractive index and the resultant phase difference

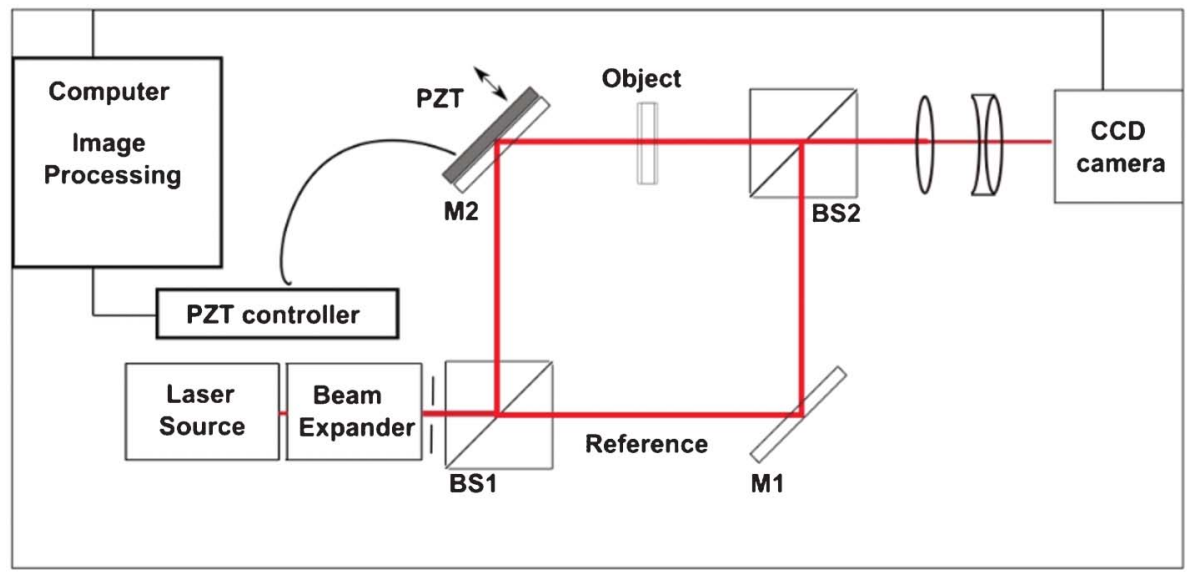

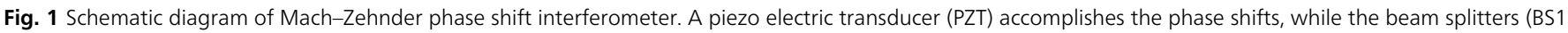
and $\mathrm{BS} 2$ ) and the mirrors (M1 and $\mathrm{M} 2$ ) are arranged in a two beam interferometer configuration. 


$$
\varphi(x, y)=\frac{2 \pi}{\lambda} \int_{0}^{d} n(x, y, z) \mathrm{d} 1 z+C
$$

where $n(x, y, z)$ is the position dependent refractive index of the solution in the growth cell, $d$ is the thickness of the cell and $\lambda$ is the wavelength of the laser light used. The constant $C$ comprises the resultant phase difference of the object and reference beam excluding the solution in the cell. Using the relation between the refractive index and the difference $\Delta c(x, y$, $z$ ) in solute concentration with respect to the equilibrium concentration, which corresponds to a refractive index $n_{\mathrm{o}}$,

$$
n(x, y, z)=n_{\mathrm{o}}+n_{1} \Delta c(x, y, z)
$$

an expression for the average solute concentration difference as a function of the phase difference is obtained

$$
\frac{1}{d} \int_{0}^{d} \Delta c(x, y, z) \mathrm{d} z=\frac{\lambda}{2 \pi n_{1}} \varphi(x, y)+D
$$

where $D$ is a constant, which can be set to zero.

A schematic diagram of the PSI is shown in Fig. 1. The whole optical setup is based upon the one that was introduced earlier by Duan and $\mathrm{Shu}^{14}$ and is mounted on a vibration-free table. A HeNe laser source of wavelength $632 \mathrm{~nm}$ is passed through a beam expander and the resultant beam diameter is controlled by a diaphragm. The beam is incident on a beam splitter (BS1) and divided into two equal beams: one functions as the reference beam and the other as the object beam. Both are then reflected by the mirrors (M1 and M2). In order to apply the consecutive phase shifts, we used an ultra-precise piezo electric transducer (S-303, Physik Instrumente (PI), Germany) (PZT) connected with a digital piezo transducer controller (E750, PI Germany). The PZT was mounted vertically at the back of M2. The controller in the "servo mode" was used such that a series of interferograms is collected by successively displacing M2 over $112 \mathrm{~nm}$ (corresponding to a phase shift of $112 \sqrt{2}$ $\mathrm{nm}=\lambda / 4$ or $\pi / 2$ ). The reference and the object beams are then superimposed by the second beam splitter (BS2) and directed through two consecutive achromatic lenses (L1 and L2) to the CCD camera (PS 4-205 GigE, Kappa). The images are recorded, digitized and processed using the MATLAB script.

\section{Results and analysis}

Dissolution of batch crystals. To validate the PSI approach, we started with dissolution experiments. A KDP seed crystal with dimensions of $0.1 \times 0.1 \times 0.7 \mathrm{~cm}^{3}$ was placed with its $c$-axis parallel to the bottom of a $0.1 \times 1 \times 4 \mathrm{~cm}^{3}$ growth cell filled with $10 \%$ or $20 \%$ undersaturated solution. A time series of concentration profiles around a KDP crystal dissolving in $20 \%$ undersaturated solution measured by PSI is shown in Fig. 2. As follows from this figure, the concentration of the solution near the dissolving crystal is higher than that of the undersaturated bulk solution far from the crystal surface. Because of its higher density, this enriched part of the solution remains at the bottom of the growth cell due to gravity and the solute transport to the rest of the solution (upwards) is governed by diffusion rather than natural convection. It can be shown that if the dissolution of a planar crystal surface is completely determined by volume diffusion, the dissolution rate, $R_{\mathrm{d}}$, decreases with time, $t$, according to

$$
R_{\mathrm{d}}(t)=\frac{D \Omega\left(c_{\mathrm{s}}-c_{\mathrm{b}}\right)}{\sqrt{\pi D t}} \propto \frac{1}{\sqrt{t}}
$$

where $D$ is the diffusion coefficient and $\Omega$ is the molecular volume of the dissolving units; $c_{\mathrm{s}}\left(\approx c_{\mathrm{eq}}\right)$ and $c_{\mathrm{b}}$ are the solute concentrations at the crystal surface and the bulk solution, respectively. The dissolution rate of the crystal is equal to the flux of the dissolving units away from its surface. This implies that the dissolution rate can be derived from the solute concentration gradient near the crystal surface, using

$$
R_{\mathrm{d}}=\left.\Omega D \frac{\mathrm{d} c}{\mathrm{~d} y}\right|_{\text {crystal surface }}
$$

where $y$ is the vertical coordinate of the growth cell.

Fig. 3 shows the solute concentration profiles perpendicular to the crystal surface as a function of time, derived from PSI measurements shown in Fig. 2. Taking the derivatives $\mathrm{d} c / \mathrm{d} y$ at the crystal surface for different times indeed shows linear dependence on the inverse of the square root of time (Fig. 3, inset). This demonstrates that the mass transport in this case is controlled primarily by diffusion. Hence, these phenomena can be successfully monitored using PSI.

Growth of batch crystals. Similar to the dissolution experiments, a tetragonal KDP crystal of dimensions $0.09 \times 0.15 \times 0.7$ $\mathrm{cm}^{3}$ was placed at the bottom of a $0.1 \times 1 \times 4 \mathrm{~cm}^{3}$ growth cell, filled with $10 \%$ or $20 \%$ supersaturated aqueous KDP solution. Due to the growing crystal, the solution becomes depleted of solute at the crystal-solution interface and by the action of gravity this lower density solution is forced upwards in the form of convection plumes (Fig. 4). Here, the mass transport growth is effectuated by natural convection and the diffusion boundary layer is thus very thin $<30 \mu \mathrm{m}$, and hardly changes in time. The convection plumes can extend up to $1 \mathrm{~cm}$ above the crystal and their reduction in supersaturation lies in the order of $2.5 \%$ of that in the bulk solution for the case of $10 \%$ supersaturation (Fig. 4, left column). The $20 \%$ supersaturated solution showed the nucleation of many crystals, which due to gravity were deposited on the crystal surface (Fig. 4, right column).

Ceiling crystal growth. Fig. 5 shows the changes of the solute concentration profile around a ceiling KDP crystal growing in $10 \%$ and $20 \%$ supersaturated solution. The dimensions of the crystal and the growth cell are the same as for the batch experiments mentioned above. In the ceiling configuration with the crystal at the top of the growth cell, the solution at the direct vicinity of the crystal again experiences a decrease in its molar concentration due to solute uptake by the growing crystal. But now, the depleted solution stays stagnant around the growing crystal by the action of gravity and the solute transport towards this ceiling crystal becomes essentially diffusion-controlled. The time dependent spatial distribution of the solute concentration perpendicular to the crystal surface is derived from the associated PSI maps. The width of the 

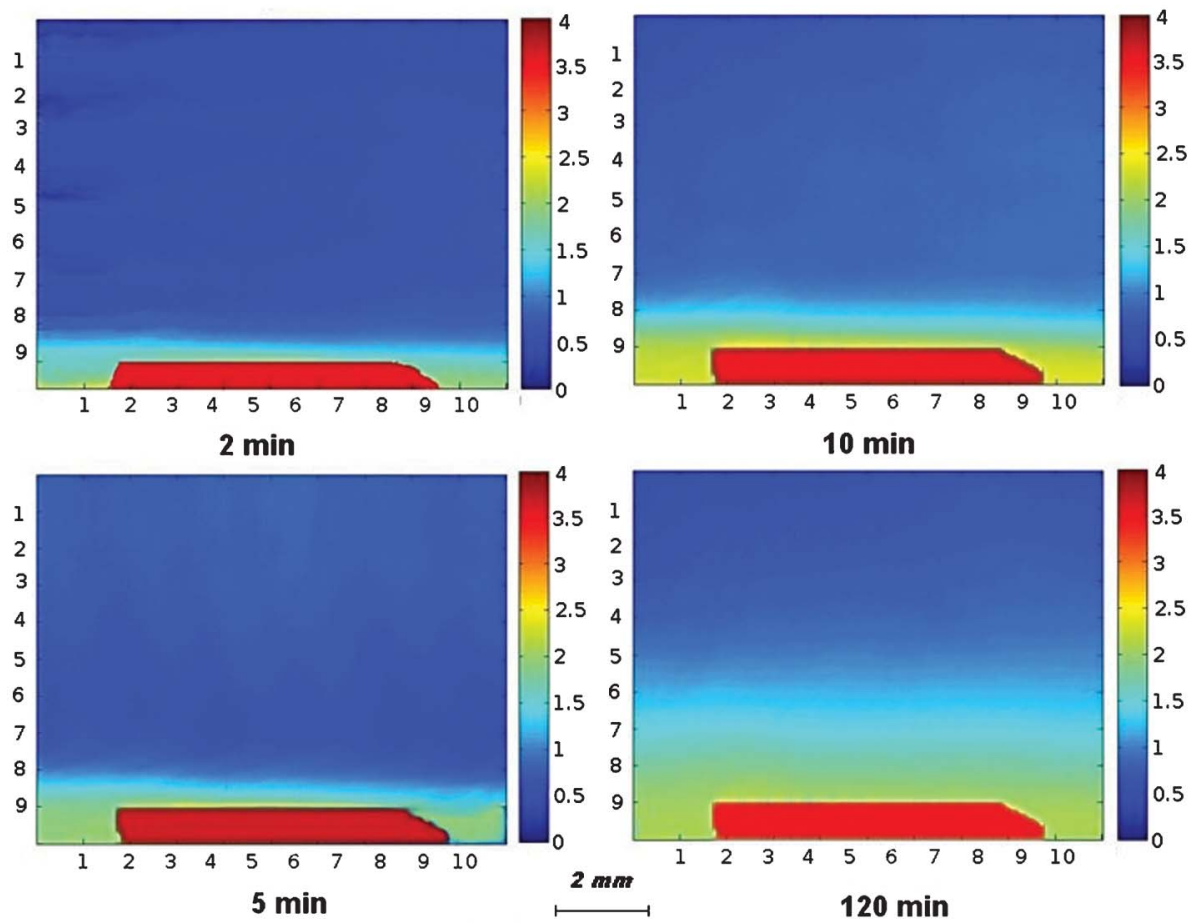

Fig. 2 The change of the concentration as a function of time for a KDP crystal in a 20\% undersaturated solution. The concentration differences are expressed in mass fractions.

depletion zone clearly increases in time (Fig. 5 and 6). The width and flow of the depletion zone $(\delta)$, as shown in the interferograms, varies directly with the concentration differ-

$$
\delta=\frac{-D}{J_{\mathrm{D}}}\left(c_{\mathrm{b}}-c_{\mathrm{s}}\right)
$$
ence, between the bulk solution and that at the crystal interface $\left(c_{\mathrm{b}}-c_{\mathrm{s}}\right)$, and inversely with the flux $\left(J_{\mathrm{D}}\right)$ such that:

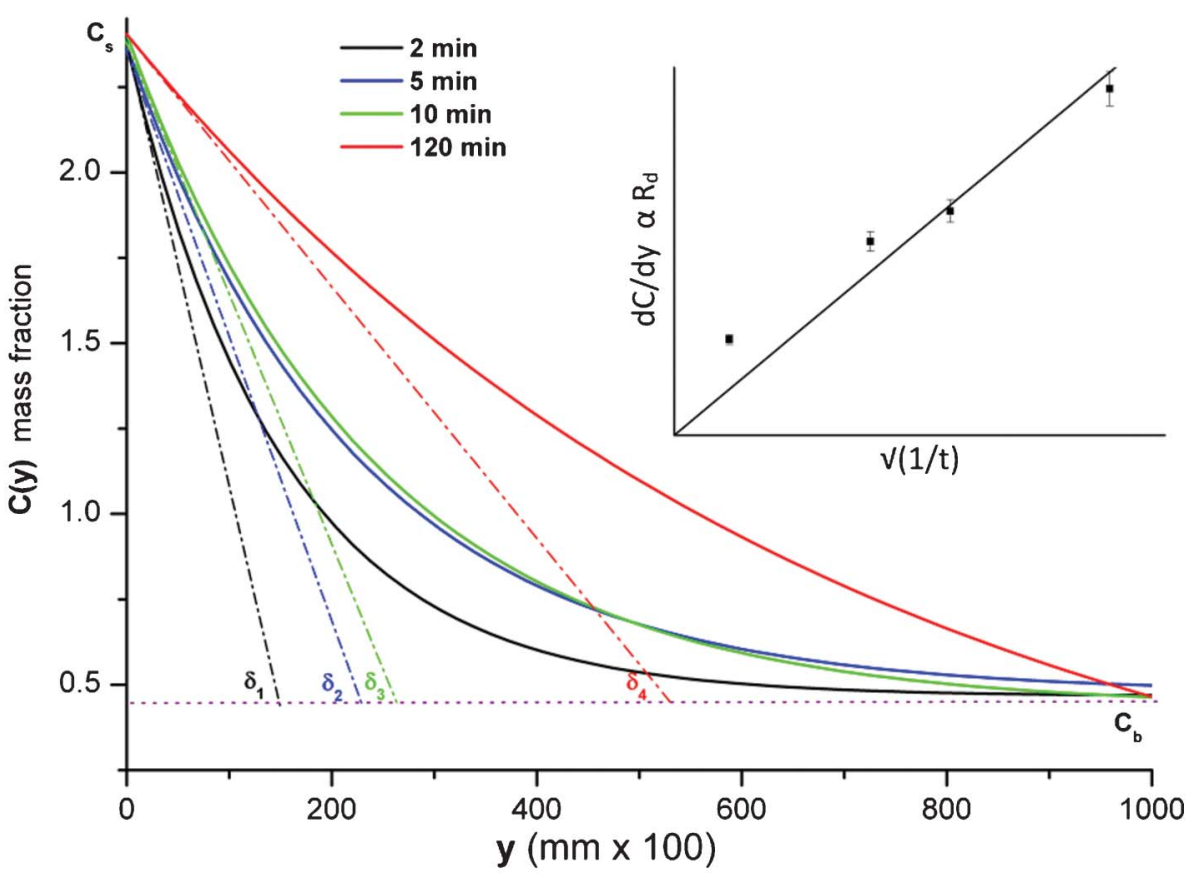

Fig. 3 The spatial distribution of concentration during the dissolution of a KDP crystal in a 20\% undersaturated solution. The upper frame shows the linear relation between the dissolution rate and the inverse of the square root of time. 

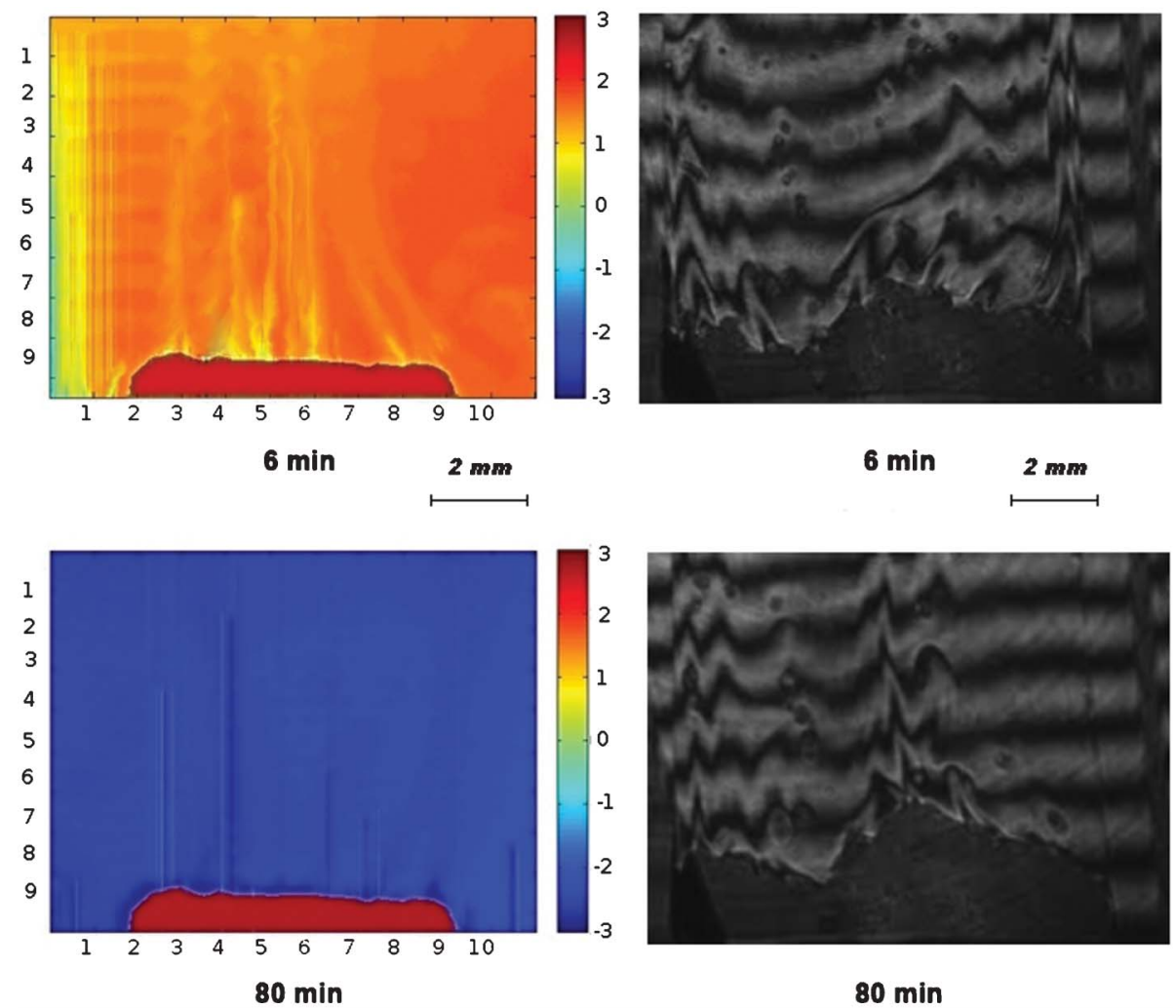

Fig. 4 Left column shows the change of the concentration profile around a batch KDP crystal growing in a 10\% supersaturated solution. The interferograms of a KDP crystal growing at $20 \%$ supersaturation are shown in the right column. Sedimentations on the crystal completely covered its surface.

This can be explained as a decrease of the flux occurring in a stagnant solution (Fig. 6, inset) with the consequence that $\delta$ extends setting mass transport to the crystal in the diffusive mode. When the oblong crystal was mounted with its $c$-axis parallel to the ceiling, the space around the crystal was filled with depleted solution within two minutes by natural convection (Fig. 5). After this stage, the diffusion layer starts to develop. This early phenomenon may account for the different spatial concentration profile of the solution near the crystal surface after two minutes (Fig. 6). This phenomenon is even more obvious if the oblong crystal is mounted to the ceiling with its long axis (the $c$-axis) perpendicular to the plane of the ceiling plate (Fig. 7). Due to the natural convection, the depleted solution flows upwards towards the ceiling and the development of the depletion zone to enclose the whole crystal took close to one hour (Fig. 7). Only then, the solute transport to the entire crystal is mediated by diffusion.

Interestingly, when we use the ceiling method to induce nucleation and crystal growth of KDP as well as a variety of proteins we always observe that the crystals nucleate and grow such that their longest axis is parallel to the plane of the ceiling plate of the growth cell. Under these conditions, when starting from small nuclei, this initial convection hardly occurs, in particular when several ceiling crystals grow simultaneously.

In the case of $20 \%$ supersaturation many crystallites nucleated at the bottom and on the side walls of the growth cell. Fortunately, the convection plumes of these crystals did not disrupt the depletion zone around the ceiling crystal. Due to convective mixing of the bulk solution, its concentration dropped significantly during the progress of the experiment (Fig. 5 and 7).

Deriving crystal growth rates from the time dependent concentration profiles in Fig. 6, using eqn (7), gives a somewhat irregular dependence as a function of $1 / \sqrt{ } t$ (Fig. 6, inset). This indicates that, in contrast to dissolution, eqn (6) is less applicable. This may be accounted for by the initial convective stage of growth, the nucleation and growth of crystallites at the bottom and the walls of the growth cell and by the influence of surface kinetics. It is well known that surface kinetics often plays a far more important role in the growth of crystals than in their dissolution.

\section{Numerical simulation}

\section{Method}

As shown in the previous section, the growth or dissolution of crystals is largely controlled by the concentration dependent solute flux at the crystal surface. This process of unit migration towards (growth) or away (dissolution) from the crystal, is described by fluid mechanics involving the concepts of continuity, flow motion and solute diffusion. ${ }^{15}$

The continuity describes the transport of a conserved quantity of fluid and can be expressed as: 
$10 \%$
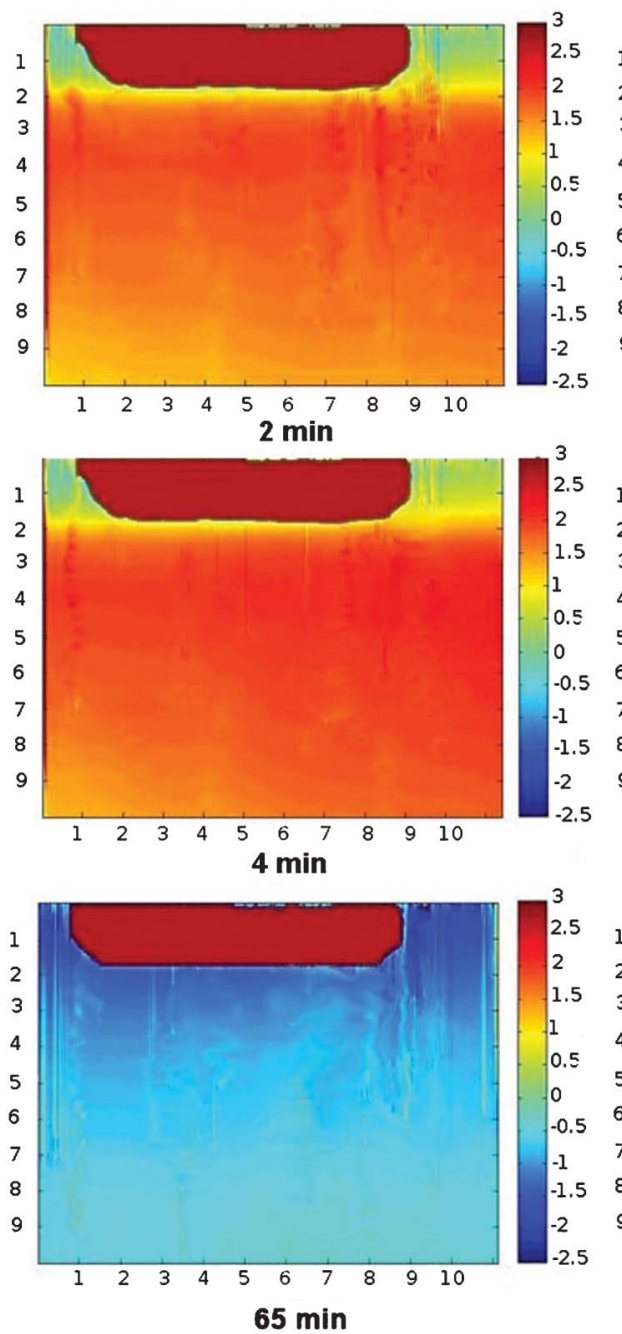

$20 \%$
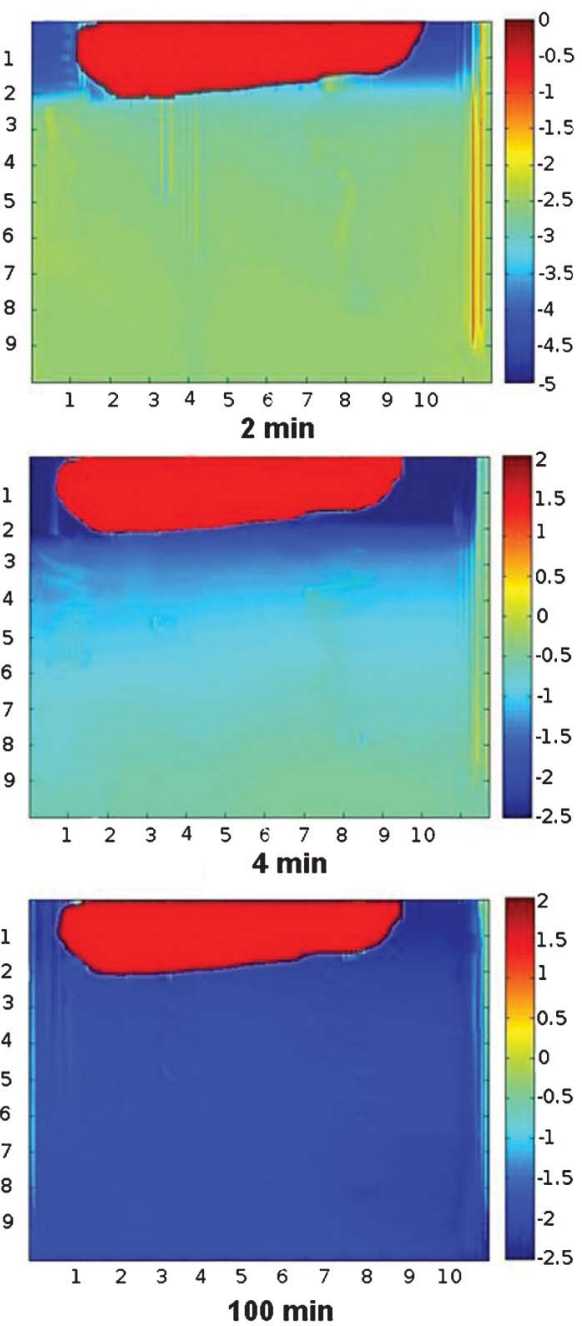

Fig. 5 The change of the concentration profile as a function of time around a ceiling KDP crystal growing in 10\% (left) and 20\% (right) supersaturated solutions.

$$
\frac{\partial^{2} \psi}{\partial y^{2}}+\frac{\partial^{2} \psi}{\partial x^{2}}+\omega=0
$$

where $\omega$ is the vorticity and $\psi$ is the potential stream function. Because our quantitative experiments were done in a thin growth cell $(1 \mathrm{~mm})$, we chose a $2 \mathrm{D}$ model and performed the calculations by using Cartesian $(x, y)$ coordinates. Combining Euler's and Navier-Stokes' equations of motion and taking their curl, the motion of the fluid can be described by the Navier-Stokes stream function vorticity equation:

$$
\frac{\partial \omega}{\partial t}-\frac{\partial \psi}{\partial x} \frac{\partial \omega}{\partial y}+\frac{\partial \psi}{\partial y} \frac{\partial \omega}{\partial x}=\mu \nabla^{2} \omega+g \frac{\partial\left(\rho-\rho_{\mathrm{o}}\right)}{\partial x}
$$

In this partial differential equation, $\mu \nabla^{2} \omega$ is the viscosity component with $\mu$ the kinematic viscosity; $g \partial\left(\rho-\rho_{\mathrm{o}}\right) / \partial x$ is defined as the force vector with $g$ the acceleration due to gravity and $\rho$ and $\rho_{\mathrm{o}}$ are the actual and the offset value of the fluid density, respectively.

Because in our case the fluid is a solution containing the growth units, diffusive solute mass transport is a function of concentration $(c)$ and local flow position such that;

$$
D \nabla^{2} c=\frac{\partial c}{\partial t}-\frac{\partial \psi}{\partial x} \frac{\partial c}{\partial y}+\frac{\partial \psi}{\partial y} \frac{\partial c}{\partial x}
$$

where $D$ is the solute diffusion coefficient.

Eqn (9)-(11) are partial differential equations, which can be solved by finite element analysis. Our aim here was to develop a numerical simulation in order to determine the stream function, vorticity and concentration for growth or dissolution processes, by providing known values for the kinematic viscosity, off-set density, gravitational acceleration, and the diffusion coefficient of the compound under study. For this, we used FlexPDE software, version 6.20, which is a multipurpose finite element analysis package capable of simulating complex physical processes. ${ }^{16}$ We applied a non-slip condition 


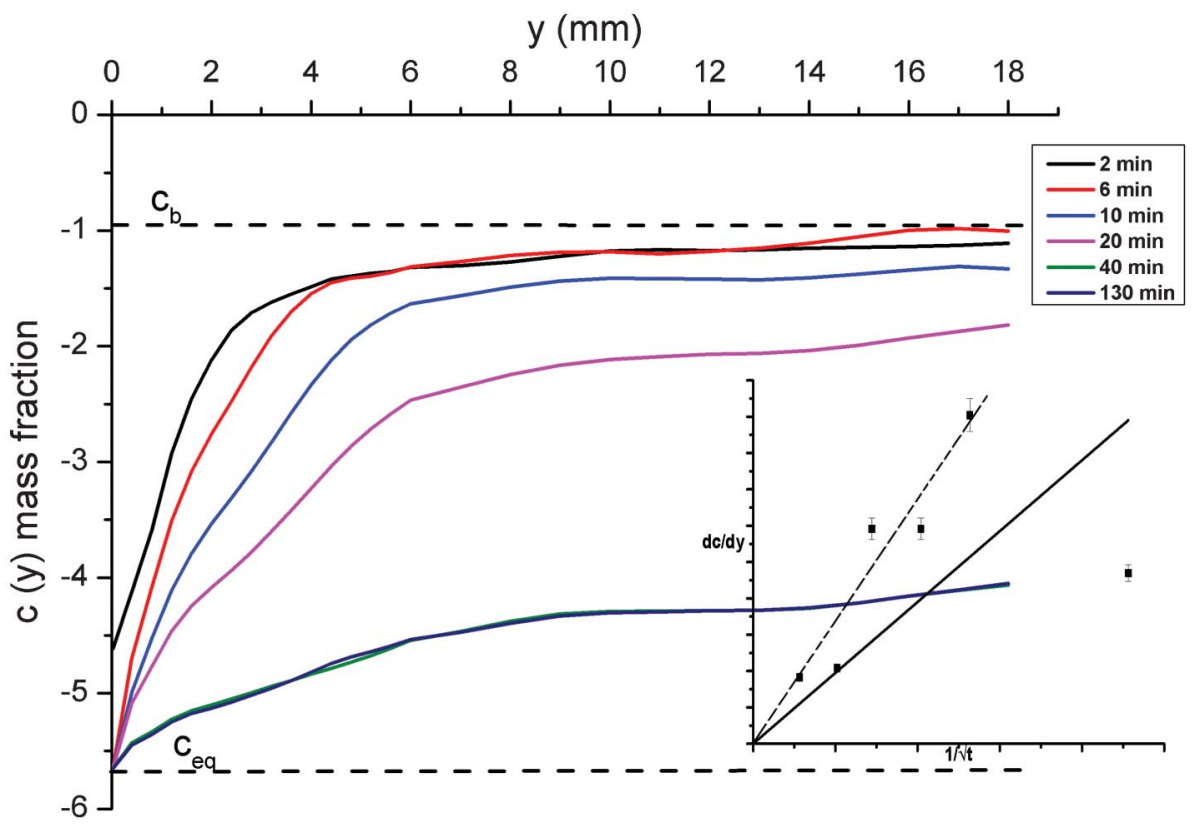

Fig. 6 The spatial distribution of the solute concentration during the ceiling growth of a KDP crystal in a 20\% supersaturated solution, The inset shows the relation between the crystal growth rate and the inverse of the square root of time. The dashed line provides a good fitting disregarding the initial convection mediated stage.

at the crystal interface and the cell walls by using a penalty method. ${ }^{17}$ The crystal was assumed to be either situated at the ceiling, the bottom or at both locations simultaneously in the growth cell. We assumed that the crystal does not show an apparent change in size during growth. The boundary conditions were defined with respect to the crystal surface and the growth cell walls such that:

i) $\Psi=0$ at the growth cell walls and crystal faces.

ii) $\frac{\partial c}{\partial n}= \begin{cases}\frac{0}{-k\left(c-c_{\mathrm{eq}}\right)} \text { at the growth cell walls } \\ - \text { at the crystal faces }\end{cases}$

In eqn (12) $k$ is the kinetic coefficient and $c$ and $c_{\text {eq }}$ are the concentrations at the crystal surface and at equilibrium, respectively. The physical constants for the compounds used in this study are given in the ESI. $\dagger$

\section{Results and analysis}

Fig. 8 shows the simulations for the ceiling growth of a downwards pointing oblong KDP crystal from a $20 \%$ supersaturated solution. There is an obvious clear agreement with the experimental data in Fig. 7. The simulated time series clearly shows the two stages in the development of the depletion layer. After 100 seconds, a thin concentration gradient normal to the crystal surface is formed by solute uptake at the crystal surface. The width of the boundary layer $(45 \mu \mathrm{m})$ is quite similar to that observed experimentally. As can be seen in the subsequent images, the depleted solution is pushed upwards by buoyant forces until the flow is prohibited any further at the "ceiling" of the growth cell. After about 5000 seconds the depleted solution encloses the whole crystal and the second stage sets in, expansion downwards by diffusionlimited mass transport.
The simulations show that the second stage of the depletion zone development, apart from a scaling factor $\left(c_{\mathrm{b}}-c_{\mathrm{eq}}\right)$, is independent of supersaturation (Fig. 9, upper row, right). This readily follows from the equation of the development of the solute concentration profile, $c(x, y, t)$, during growth in a one dimensional system limited by mass transport:

$$
c(x, y, t)=-\left(c_{b}-c_{e q}\right) \operatorname{erfc}[y / 2 \sqrt{D t}]+c_{b}
$$

where erfc is the complementary error function. In contrast, the dissolution of a ceiling crystal is governed by natural convection, leading to a thin boundary layer around the crystal and a downward directed convection plume. As seen in Fig. 9 (upper row, left) the diffusion boundary layer gets thinner for increasing undersaturation.

Because in practice it is very difficult to avoid nucleation at the bottom of the growth cell, in particular at high supersaturation, which is the optimal condition for most protein crystallization protocols, we also simulated the condition where two crystals grow or dissolve at both locations simultaneously (Fig. 9, lower row). At higher supersaturation, the developed convection plumes are able to reach the depletion zone around the ceiling crystal, still the latter is hardly distorted. In the presence of more batch and ceiling crystals the solution depletes faster, but the overall mechanism remains the same. Another effect that can be easily spotted is that the concentration of the bulk solution decreases rapidly, due to the faster growth of the batch crystals and the continuous mixing of the solution by the action of natural convection. As a consequence, the depletion zone of the ceiling crystals, despite being intact, fades away in the course of time. This was also observed in our KDP growth experiments (Fig. 7). The simulation of two crystals simulta- 

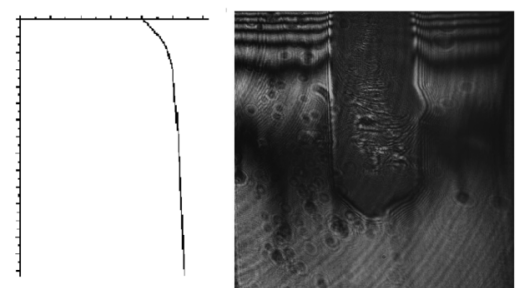

$3 \mathrm{~min}$
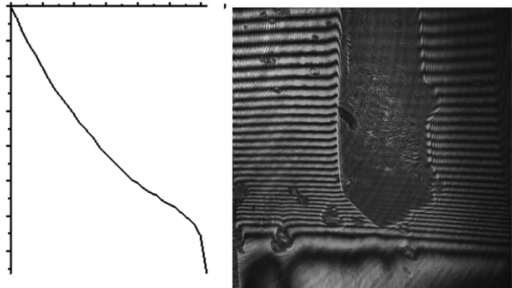

57 min
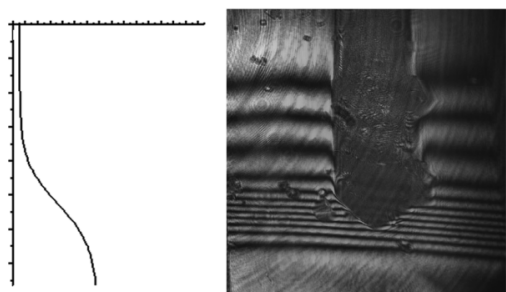

$167 \mathrm{~min}$

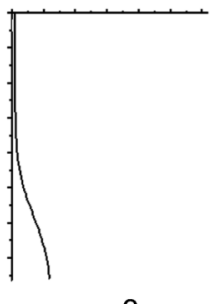

$3 \mathrm{~mm}$

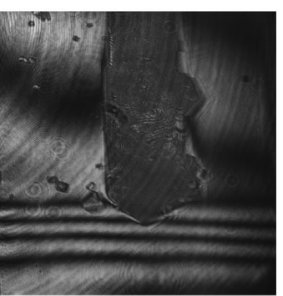

$307 \mathrm{~min}$

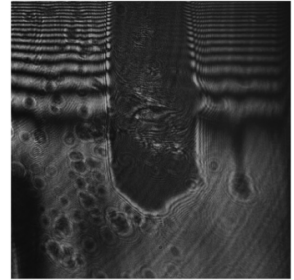

8 min

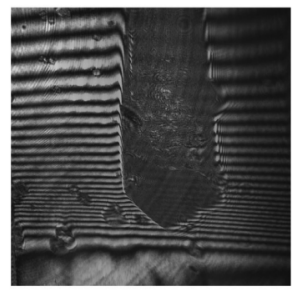

77 min

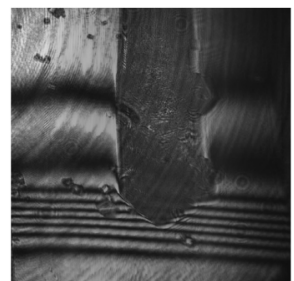

$187 \mathrm{~min}$

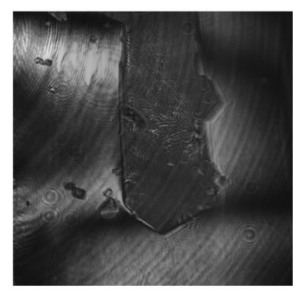

$385 \mathrm{~min}$

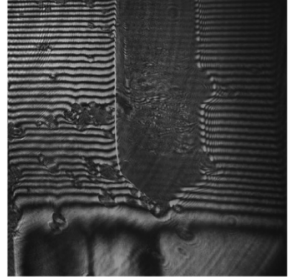

$27 \mathrm{~min}$

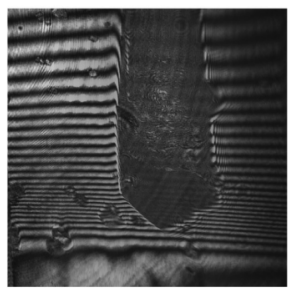

$107 \mathrm{~min}$

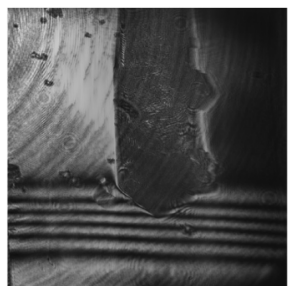

$227 \mathrm{~min}$

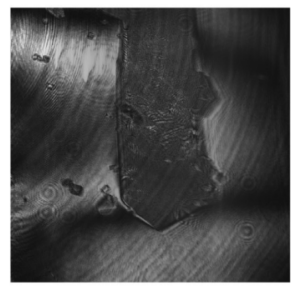

$690 \mathrm{~min}$

Fig. 7 Timelines of the interferograms of ceiling KDP crystals which extends deeply in the growth solution and grows in $20 \%$ supersaturated solutions. The

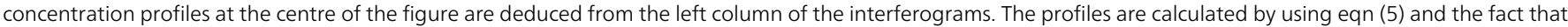
the distance between two interference lines corresponds to a phase difference of $\phi=2 \pi$.

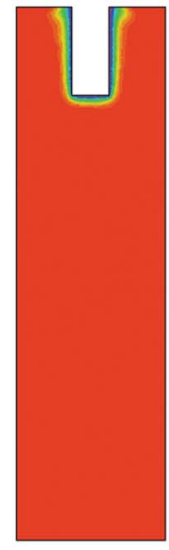

100

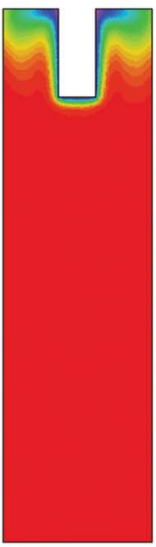

500

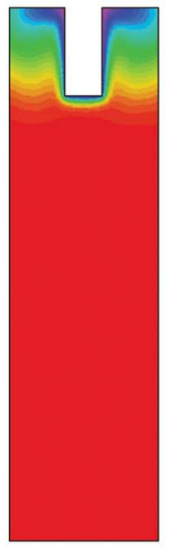

1000

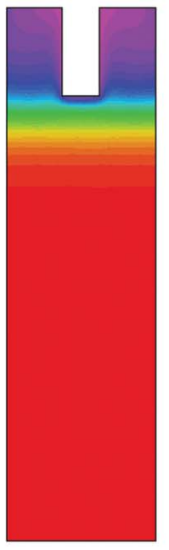

5000

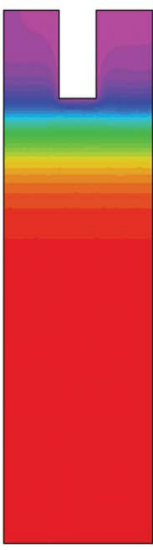

10,000

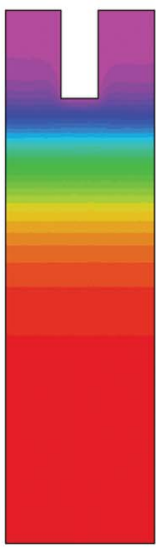

25,000

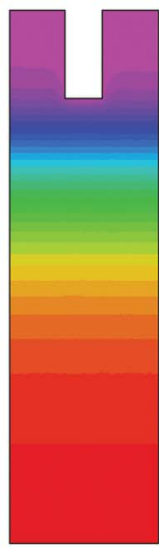

50,000

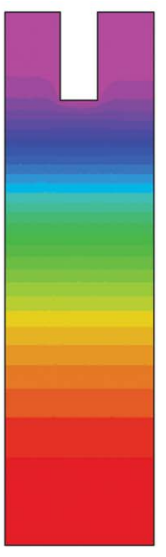

100,000

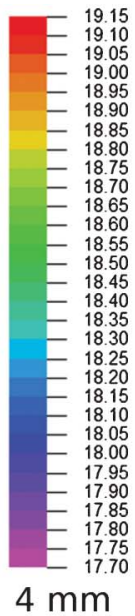

$4 \mathrm{~mm}$

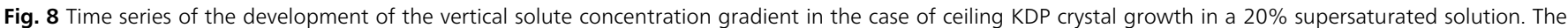
calibration bar accounts to the concentration profile (in $\mathrm{gm} \%$ ) and the time is counted in seconds after the initiation of growth. 

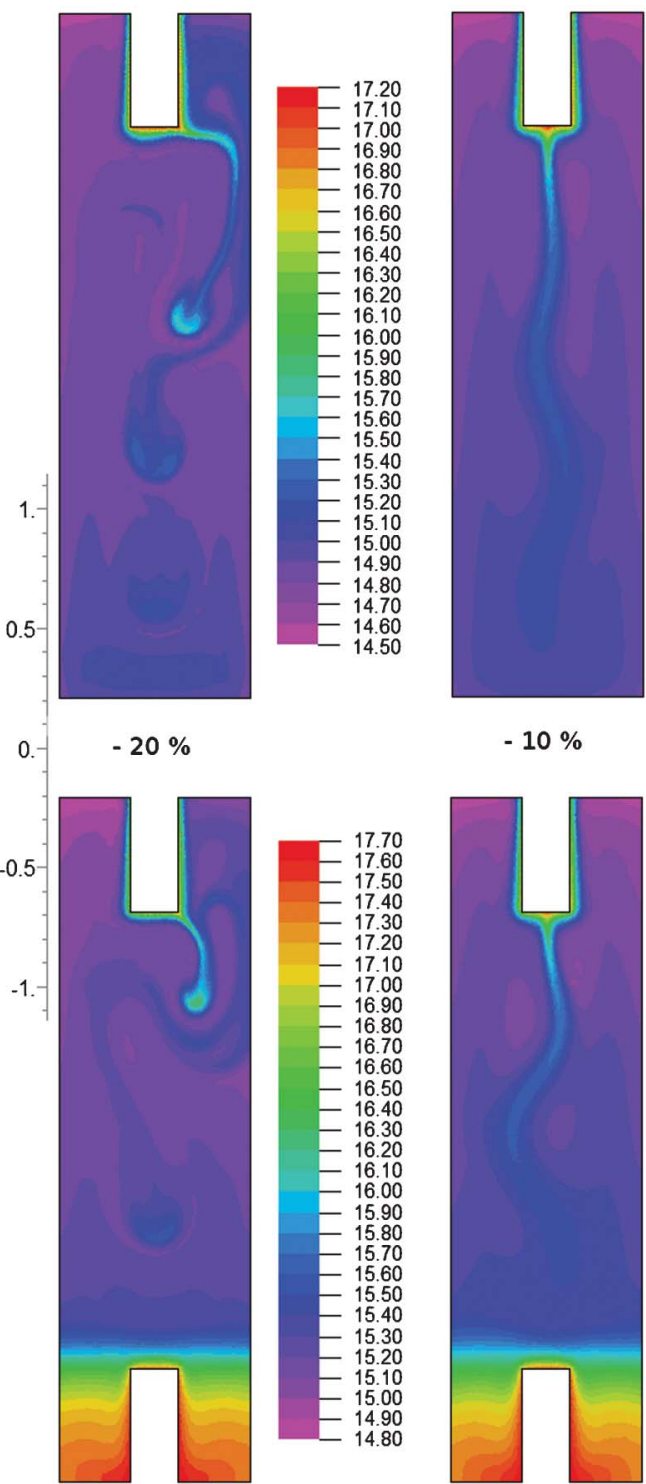

$-10 \%$

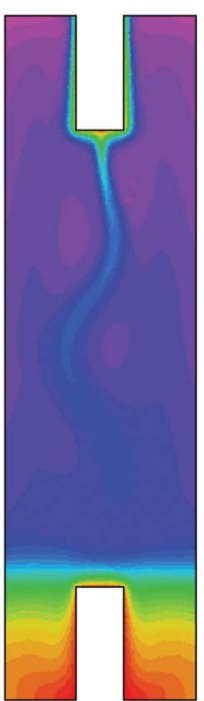

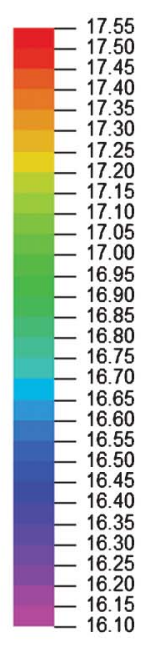
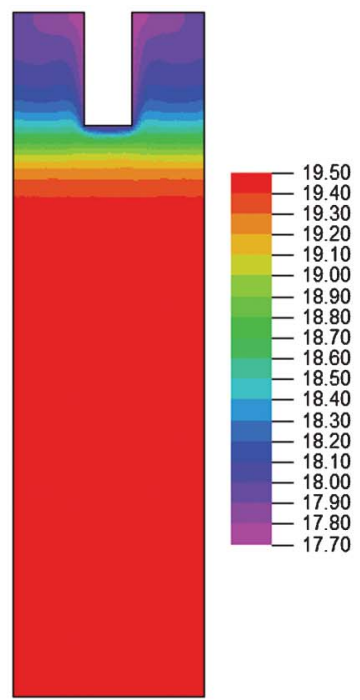

$+10 \%$
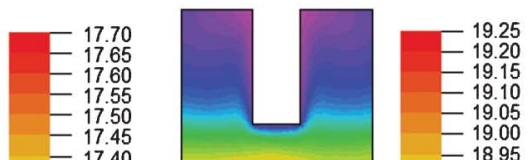

19.25
-19.20
-19.15
-19.10
-19.05
-19.00
-18.95

-18.95
-18.90
-18.85

$-18.90$

$\begin{array}{r}18.80 \\ -1875 \\ \hline\end{array}$

$\begin{array}{r}-18.75 \\ -18.70 \\ \hline\end{array}$

$-18.65$

18.60
-1855
-18850

-18.55
-18.50
-18.45

18.45
-18.40
-18.35

$-18.35$

$\begin{array}{r}18.35 \\ -18.30 \\ \hline\end{array}$

18.25
-1820

$\begin{array}{r}18.20 \\ -18.15 \\ \hline\end{array}$

$\begin{array}{r}18.15 \\ -18.10 \\ \hline\end{array}$

$-18.05$

$-18.00$

18.00
-17.95
-17.90

$\begin{array}{r}17.90 \\ -17.85 \\ \hline\end{array}$

$-17.80$

17.85
-17.75
-1770
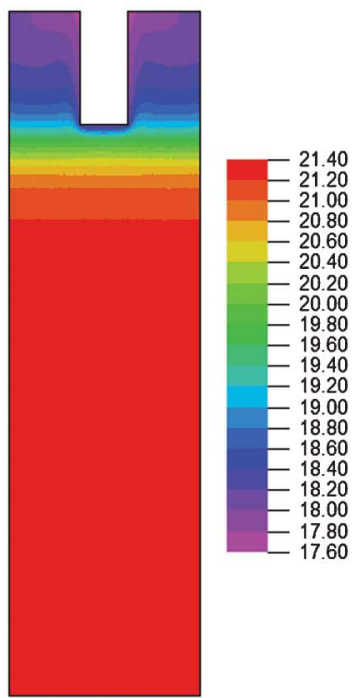

$+20 \%$
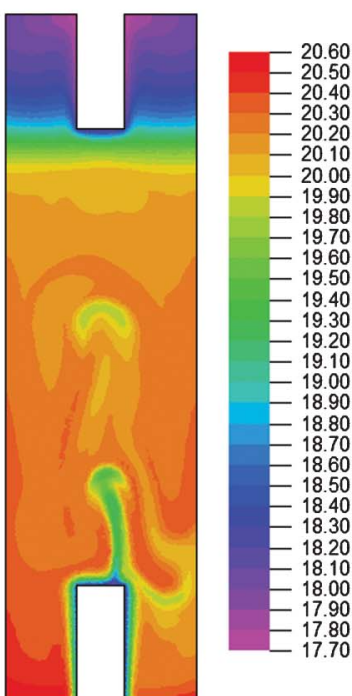

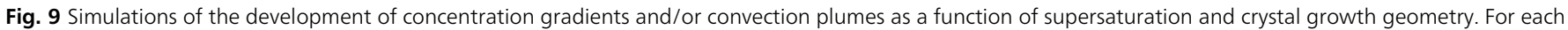
separate simulation, the resultant concentration profile after one hour of growth or dissolution is presented along with its associated legend (KDP in $\mathrm{g} \%$ ).

neously dissolving at the top and bottom of the cell yielded the same results, but in opposite directions (Fig. 9, lower row, left).

Among the factors that affect the growth process are the intrinsic properties of the solutes (molecule to be crystallized, buffer, salts, precipitant) and the solvent used to prepare the crystallization solution. One of these properties, the diffusion coefficient of the molecule to be crystallized, has the highest influence on the shape, progression and extension of the concentration profiles. Fig. 10 displays simulations of the concentration profiles for three different compounds; sodium chlorate $\left(\mathrm{NaClO}_{3}\right), \mathrm{KDP}$ and HEWL. The diffusion coefficients $D$ of these compounds are $1.5 \times 10^{-5}, 6.7 \times 10^{-6}$, and $1.0 \times$ $10^{-6} \mathrm{~cm}^{2} \mathrm{~s}^{-1}$, respectively. ${ }^{7,18}$ The decrease of these values for the three compounds is directly related to the decrease of the progression of the depleted zone and therefore, the growth
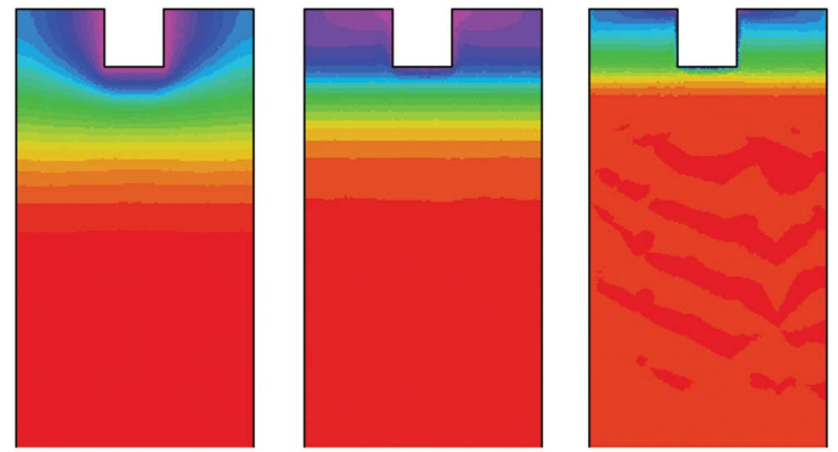

Fig. 10 Simulated concentration profiles of (from left) $\mathrm{NaClO}_{3}, \mathrm{KDP}$ and HEWL ceiling crystals one hour after the initiation of growth. The figure displays only a magnified view for the upper part of the growth cell (which is $1 \times 4 \mathrm{~cm}^{2}$ ). 
rate of the three types of crystals as follows from eqn (5) and (11). Experimentally, at the same starting supersaturation under diffusion-limited crystal growth conditions, the final crystal size of an average HEWL crystal is about five times smaller than that of KDP, which again is smaller than $\mathrm{NaClO}_{3}$. In our simulations, starting from the same size of seeding crystals, the development of the depletion zone is the slowest for HEWL and the fastest for $\mathrm{NaClO}_{3}$, in accordance with their crystal growth rate and diffusion coefficient. This rate of development can be enhanced by the nucleation of several crystals at the ceiling of the growth cell as discussed below, which generally is the case for ceiling growth. In comparison with other proteins, HEWL has one of the highest values for its diffusion coefficient (e.g. BSA $=6.09 \times 10^{-7} \mathrm{~cm}^{2} \mathrm{~s}^{-1}$, bovine insulin $\left.=7.3 \times 10^{-7} \mathrm{~cm}^{2} \mathrm{~s}^{-1}\right)$. This makes the method broadly applicable as long as certain dimensional ratios are preserved, as discussed below. In such a case, the size of the growth cells can be much reduced and still the buoyancy-assisted diffusion and the depleted zone of the ceiling crystals will not be disturbed by the convection plumes rising from any batch crystals which happen to grow at the bottom.

\section{Dimensions of the growth cell}

As follows from the experimental and simulation results, successful ceiling crystallization depends on many factors. One of the most important points for protein crystallographers is the achievable sample volume per trial. Nowadays, many commercially available crystallization set-ups adopt vapour diffusion and micro-batch platforms in the nano- to microliter range for both globular and membrane proteins. The ceiling method is not suitable for broad initial screening of crystallization conditions, because of the minimal volume requirement of about $10 \mu \mathrm{l}$ per trial, but rather for improving crystal quality under preselected conditions. In this context it is important to consider the dimensions of the growth cells, in order to achieve diffusion-limited mass transport. Experimentally, we examined this by performing two sets of trials using differently sized growth cells of $7 \mathrm{~mm}$ radius and 2 $\mathrm{mm}$ height (group 1) and $3.5 \mathrm{~mm}$ radius and $2 \mathrm{~mm}$ height (group 2) in which tetragonal HEWL crystals were grown. The effect of adding impurities with different segregation coefficients $(k)^{19}$ to the mother liquor, i.e. bovine insulin and bovine serum albumin (BSA), was also studied. As shown in Fig. 11, the ceiling crystals grown in the narrower vials (right column) in all cases are morphologically defect-free when compared to their counterparts in the wider vials (left column) and also are hardly affected by any impurities present $[k<1]$. Similar results were reported earlier in a space microgravity experiment. ${ }^{20}$ In addition, preliminary SDS PAGE analysis indeed confirmed the optical data, indicating lower incorporation of BSA in ceiling crystals grown in the narrower cells (not shown). This demonstrates that the ceiling method succeeds to accomplish a similar diffusion-limited growth conditions.

In order to understand this effect of the vessel width, we simulated the growth cells by considering crystals on the
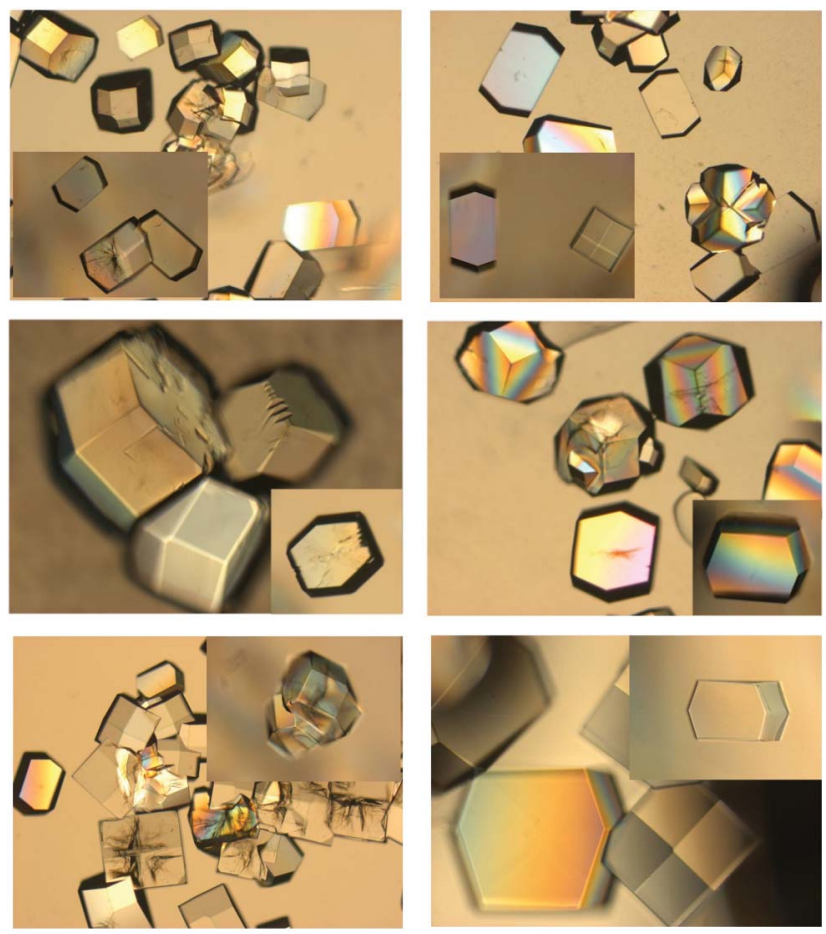

Fig. 11 Ceiling (insets) and batch HEWL crystals grown in wide (left column, top) and narrow (right column, top) growth cells. The second and third rows display HEWL crystals grown in the presence of bovine insulin and BSA as an impurity, respectively. Often ceiling crystals were fewer. All images are displayed at the same scale. Pictures are representative for the entire population.

ceiling and at the bottom simultaneously (Fig. 12). Obviously, the depth of the depletion zone varies inversely with the width of the growth cell at constant height of the growth cell. This probably is because it takes longer time in the wider cell to fill the horizontal layers with depleted solution and hence to start diffusion limited growth.

At the early convection mediated stage, the probability of impurity incorporation is relatively high. Therefore, regardless of the value of the segregation coefficient, the impurities will find their way to the tetragonal HEWL crystal nuclei (in case of BSA), inducing more nucleation and twinning, or during crystal growth (in case of bovine insulin) leading to crystals possessing rougher surfaces. Thus, we surmise that rapid enclosure of growing ceiling crystals by the depletion zone will result in better quality crystals. Further, we anticipate that this will be enhanced by simultaneous growth of a relatively large number of ceiling crystals and by a not too small ratio of the height of the growth cell relative to its width (e.g. in the order of 3 to 4 , as used routinely and also in accordance with the 10 $\mu \mathrm{l}$ nanoplate wells). This was indeed borne out by further simulations, showing that the presence of many nucleation sites improves the solute depletion pattern of every individual ceiling crystal (not shown). We also simulated a nanoplate well, and although it provides limited space for nucleation, it does show rapid development and extension of the depletion 
(a)
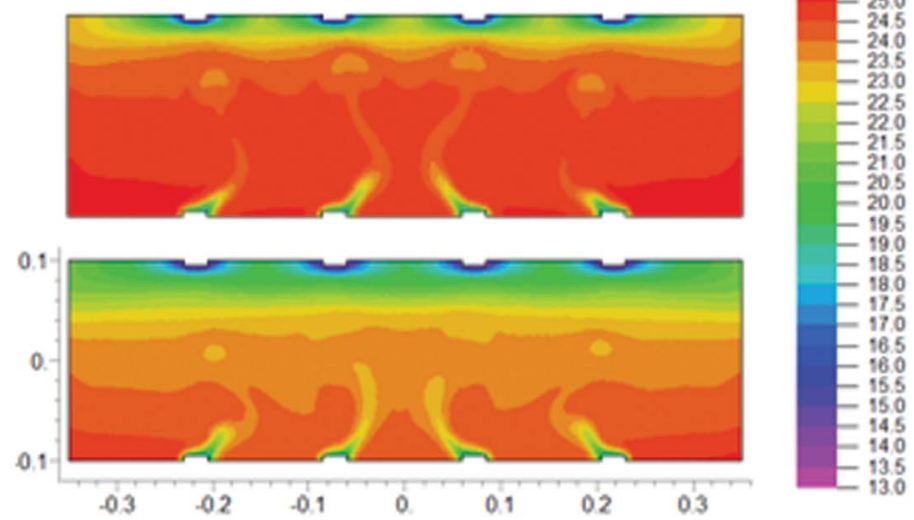

(b) (c)

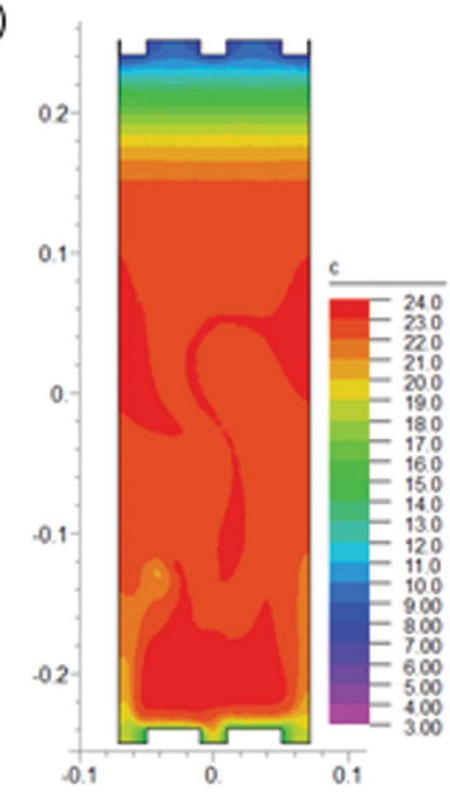

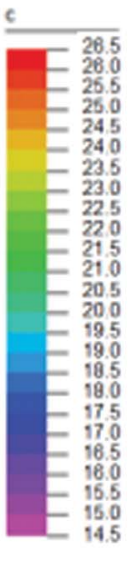

Fig. 12 Numerical simulations of HEWL crystal growth in cells with dimensions mimicking those in Fig. 11 and of a $10 \mu l$ well in a nanoplate. After 10 min, the

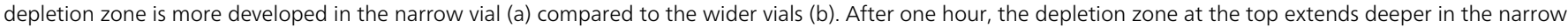

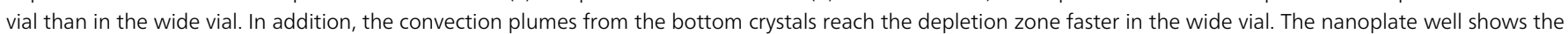
best development of the depletion zone (c) (scale in $\mathrm{cm}$, concentrations in $\mathrm{mg} \mathrm{ml}^{-1}$ ).

zone downwards (Fig. 12), confirming that the ceiling method is also applicable to this very small volume scale.

\section{Experimental procedure}

The concentration profile development during ceiling and batch growth/dissolution was investigated by experiments using a Mach-Zehnder phase shifting interferometer. KDP $\left(\mathrm{KH}_{2} \mathrm{PO}_{4}\right.$, Merck) was used as a model compound in this study. Quartz cells of high optical quality, $1 \times 1 \times 4 \mathrm{~cm}^{3}$ and $0.1 \times 1$ $\times 4 \mathrm{~cm}^{3}$ in size (Hellma Analytics, Germany) were used as growth cells in the PSI experiments. KDP seed crystals were mounted with vaseline (Sigma-Aldrich) in the cell with their long $c$-axis either parallel or perpendicular to the plane of the cell top or bottom. Four KDP solutions were prepared to achieve concentration levels of $20 \%$ and $10 \%$ below saturation and levels of $10 \%$ and $20 \%$ above saturation, respectively. The cells were completely filled with the appropriate solution and sealed using a small film of vaseline and glass cover slip. After preparation, the growth cell was placed in the object beam optical path of the interferometer.

HEWL (lot\# L6876), bovine insulin (lot\# 069k09822) and BSA (lot\# 85040C) were purchased from Sigma-Aldrich and used without further purification. Other inorganic salts were also purchased from Sigma-Aldrich at the best grade available. The tetragonal HEWL crystals were grown from $20 \mathrm{mg} \mathrm{ml}^{-1}$ HEWL and $35 \mathrm{mg} \mathrm{ml}^{-1} \mathrm{NaCl}$ as a precipitant in $0.1 \mathrm{M}$ sodium acetate buffer adjusted to $\mathrm{pH} 4.5$ using acetic acid.

The effect of different impurities on the protein crystal growth of HEWL was investigated by adding either bovine insulin or bovine serum albumin (BSA), to final concentrations of $0.2 \mathrm{mg} \mathrm{ml}^{-1}$ and $6 \mathrm{mg} \mathrm{ml}^{-1}$, respectively.

All experiments were conducted in a temperature-controlled room set at $20{ }^{\circ} \mathrm{C}$. 


\section{Conclusions}

We studied the solute concentration profiles developing during growth as well as dissolution of crystals mounted at the top (ceiling method) and/or at the bottom of the growth cell (batch method). Through a Mach-Zehnder phase shifting interferometer (PSI) and a five-frame algorithm, we successfully monitored these profiles which develop during dissolution and growth of KDP crystals. The PSI data are substantiated by finite element based numerical simulation.

The profiles resulting from dissolving batch crystals showed a morphological resemblance to that from growing ceiling crystals. In both cases, after an initial period of formation of an expanding enrichment/depletion zone (governed by natural convection), the process becomes largely controlled by diffusion-limited solute transport. Yet, the observed differences between dissolution and growth provide evidence that in the latter case the surface kinetics and, at higher supersaturation, the nucleation of crystallites play a non-negligible role.

In addition, the growth of ceiling HEWL crystals and the simulation of this process reveal that the cell dimensions are critical for effective ceiling crystallization. At a low height to width ratio, diffusion controlled mass transport develops slower, giving more chances for the rising convection plumes to perturb the growth of ceiling crystals and thus making the merits of the method less favorable. The simulations also showed that the ceiling method can be effective at a very small scale, given that the cell aspect ratios are such that the initial convection stage is as short as possible.

In short, our investigations generally confirm that the ceiling approach affords a simple and economical set-up to realize fully convection-free mass transport and therefore diffusion-limited crystal growth.

Extrapolation of the finite element simulation to cylindrical coordinates and using moving boundary conditions in order to expand this analysis to the nucleation step and impurity incorporation are in progress.

\section{Acknowledgements}

The authors would like to sincerely thank Wil Corbeek, Jan van Kessel, Wiesiek Szweryn and Erik de Ronde for the technical support. This project is funded by The Netherlands Foundation for Scientific Research through its Chemical Council (NWO-CW, project 700.57.022).

\section{Notes and references}

1 A. A. Chernov and E. I. Givargizov, Modern Crystallography III: Crystal Growth, Springer-Verlag, Berlin, 1984.

2 A. McPherson, Crystallization of Biological Macromolecules, Cold Spring Harbor Laboratory Press, New York, 1999.

3 J. M. García-Ruiz, Key Eng. Mater., 1991, 58, 87-106.

4 P. W. G. Poodt, M. C. R. Heijna, P. C. M. Christianen, W. J. P. van Enckevort, W. J. de Grip, K. Tsukamoto, J. C. Maan and E. Vlieg, Cryst. Growth Des., 2006, 6, 2275-2280.

5 A. McPherson, A. Greenwood and J. Day, Adv. Space Res., 1991, 11, 343-356.

6 H. Lin, D. N. Petsev, S. T. Yau, B. R. Thomas and P. G. Vekilov, Cryst. Growth Des., 2001, 1, 73-79.

7 P. W. G. Poodt, M. C. R. Heijna, A. Schouten, P. Gros, W. J. P. van Enckevort and E. Vlieg, Cryst. Growth Des., 2009, 9, 885-888.

8 A. Adawy, S. Törnroth-Horsefield, W. J. De Grip, W. Van Enckevort and E. Vlieg, Cryst. Growth Des., 2012, DOI: 10.1021/cg301497t.

9 G. Dhanaraj, K. Byrappa, V. Prasad and M. Dudley, Springer Handbook of Crystal Growth, 2010, 759-794.

10 F. A. Jenkins and H. E. White, in Fundamentals of Optics, McGraw-Hill, Inc., 1976.

11 S. Verma and P. J. Shlichta, Prog. Cryst. Growth Charact. Mater., 2008, 54, 1-120.

12 A. V. Lugt, IEEE Trans. Inf. Theory, 1964, 10, 139-145.

13 J. Schmit and K. Creath, Appl. Opt., 1995, 34, 3610-3619.

14 L. Duan and J. Shu, J. Cryst. Growth, 2001, 223, 181-188.

15 T. Kambe, Elementary Fluid Mechanics, World Scientific Pub Co Inc., 2007.

16 T. FlexPDE, PDESolutions Inc., http: ||www.pdesolutions. com.

17 F. C. G. De Marco, C. R. De Andrade and E. L. Zaparoli, Int. Commun. Heat Mass Transfer, 2003, 30, 495-504.

18 P. W. G. Poodt, M. C. R. Heijna, P. C. M. Christianen, W. J. P. van Enckevort, J. C. Maan and E. Vlieg, Cryst. Growth Des., 2008, 8, 2200-2204.

19 R. A. Judge, E. L. Forsythe and M. L. Pusey, Biotechnol. Bioeng., 1998, 59, 776-785.

20 B. R. Thomas, A. A. Chernov, P. G. Vekilov and D. C. Carter, J. Cryst. Growth, 2000, 211, 149-156. 